\title{
Proximal Medial Tibial Bone Graft Harvesting in Foot and Ankle Surgery
}

${ }^{1}$ Rakesh Dalal, ${ }^{2}$ Hari Kovilazhikathu Sugathan, ${ }^{3}$ Rajesh Rachha

\begin{abstract}
Cancellous bone graft harvesting from proximal tibia is usually by a lateral approach. We describe our technique and results in harvesting proximal tibia bone graft by a medial approach in foot and ankle surgery. Our results confirm that medial proximal tibial bone graft harvesting is a relatively safe and easy procedure to obtain adequate amount of autogenous cancellous bone graft. Donor site morbidity was found to be very low and fusion rate was found to be excellent in a variety of foot and ankle surgeries.
\end{abstract}

Keywords: Bone graft harvesting, Foot and ankle, Proximal medial tibia.

How to cite this article: Dalal R, Sugathan HK, Rachha R. Proximal Medial Tibial Bone Graft Harvesting in Foot and Ankle Surgery. J Foot Ankle Surg (Asia-Pacific) 2016;3(1):6-9.

\section{Source of support: Nil}

\section{Conflict of interest: None}

\section{INTRODUCTION}

Cancellous bone graft is needed in small and moderate amounts in many procedures in foot and ankle surgery. Such graft can be obtained from the iliac crest, proximal tibia, distal tibia, and calcaneum. Iliac crest graft is associated with significant donor site morbidity. Exposure is difficult for distal tibial graft harvest. Calcaneal graft is limited in quantity. ${ }^{1}$ Synthetic bone graft substitutes and allografts have unproven efficacy and have obvious cost implications. Proximal tibial graft can be harvested from either the medial or lateral cortical surface. Medial tibial exposure is easier, quicker, and involves virtually no soft tissue dissection. We present our surgical technique and results of medial proximal tibial bone graft harvesting (MPTBGH).

\footnotetext{
${ }^{1}$ Consultant, ${ }^{2}$ Fellow, ${ }^{3}$ Senior Clinical Fellow

1,2Department of Trauma and Orthopaedics, Stepping Hill Hospital, Cheshire, United Kingdom

${ }^{3}$ Department of Trauma and Orthopaedics, Leicester General Hospital, Leicestershire, United Kingdom
}

Corresponding Author: Rakesh Dalal, Consultant, Department of Trauma and Orthopaedics, Stepping Hill Hospital, Cheshire United Kingdom, Phone: 01614831010, e-mail: rdalal7119@ aol.com

\section{Surgical Technique}

Cancellous bone graft was harvested from ipsilateral proximal tibia through medial approach. Patient supine, tibial tuberosity, and anterior and posterior borders of medial surface marked. Skin incision is about 5 to $7 \mathrm{~cm}$, along the midpoint of anterior and posterior borders as marked (Fig. 1). Periosteum is incised over a $6 \times 5 \mathrm{~cm}$ area and reflected with a periosteum elevator. Drill holes are made at $1 \mathrm{~cm}$ intervals, averaging six longitudinally and four to five horizontally to complete a rectangle (Fig. 2). The holes are joined together with a saw blade/osteotomes to raise a rectangular, bevelled window (Fig. 3) of cortical bone and is lifted out and saved carefully (Fig. 4).

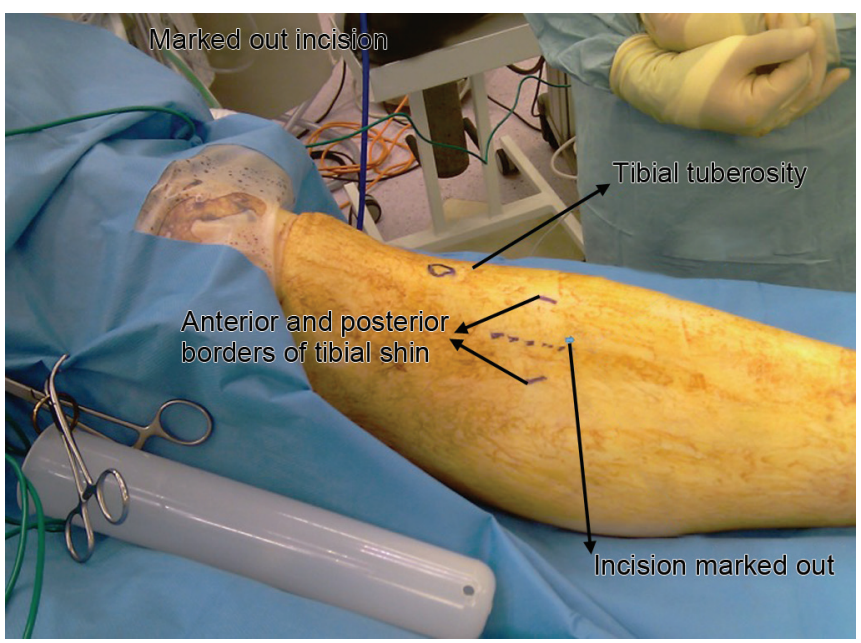

Fig. 1: Skin incision

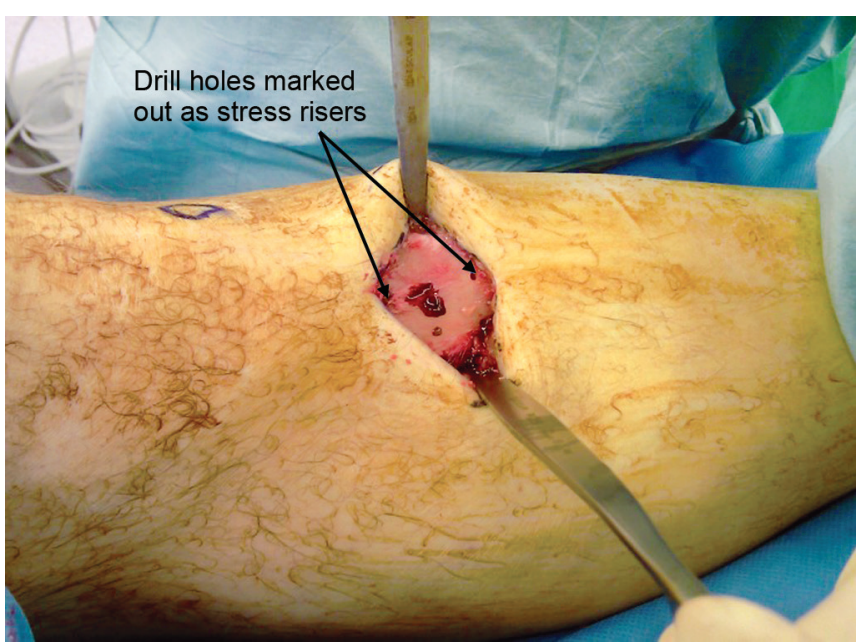

Fig. 2: Drill holes 
Cancellous bone is then harvested from the medullary cavity using a curette and nibbler. Good quantity of graft is usually available from the metaphyseal bone (Fig. 5). Marrow is harvested into a syringe using a "quill" (Fig. 6)

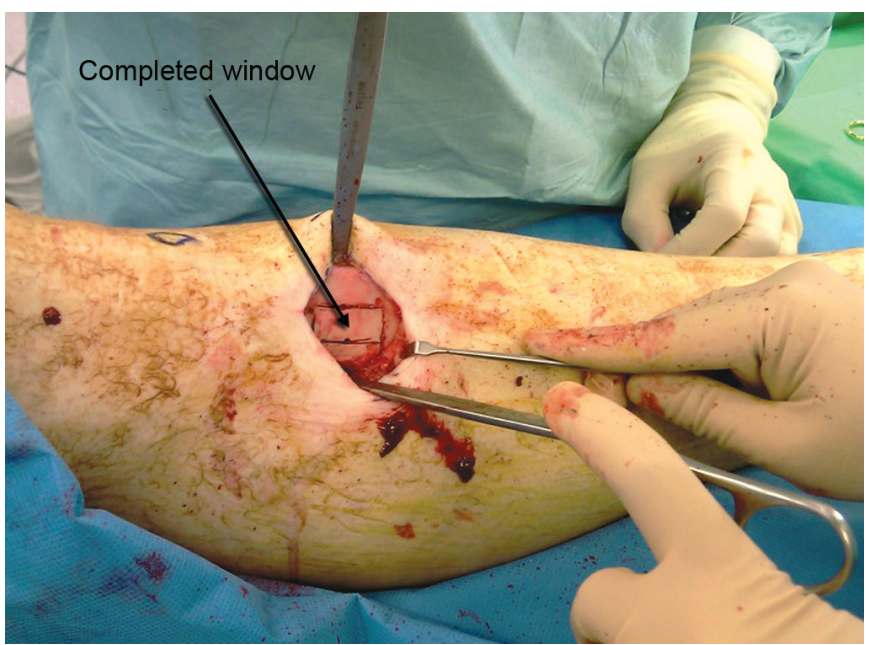

Fig. 3: Cortical window

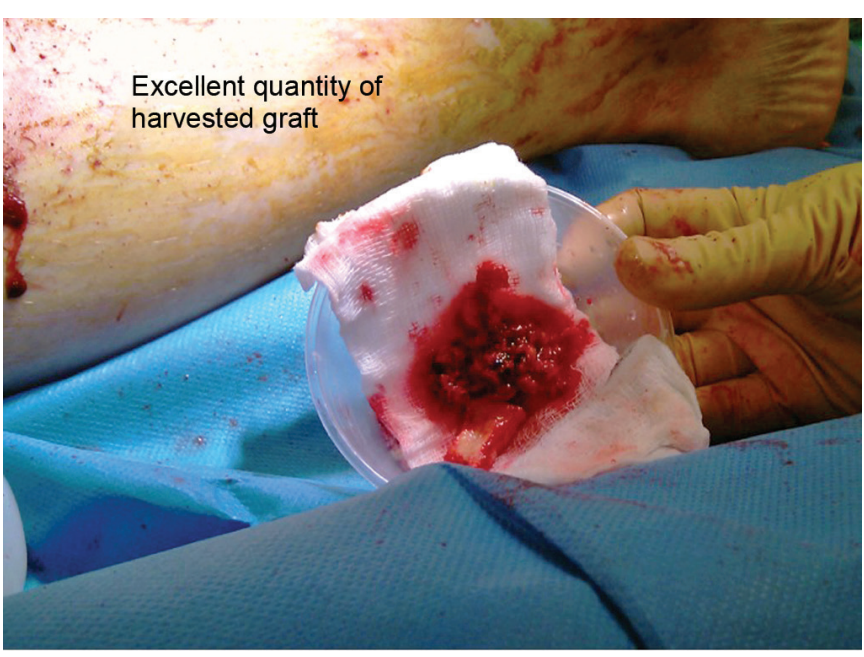

Fig. 5: Graft quantity

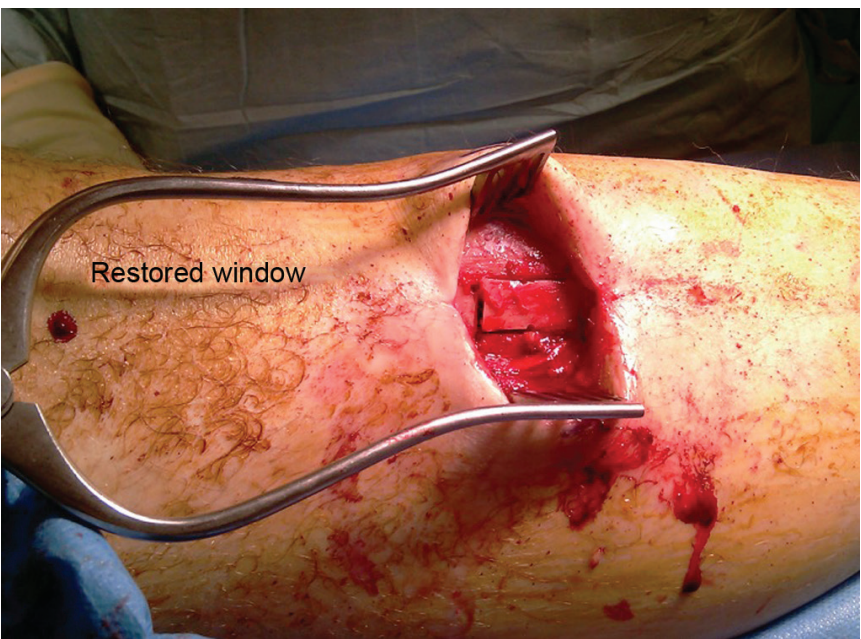

Fig. 7: Window repositioned and is injected into the recipient site to improve the osteogenic potential. The window is then repositioned (Fig. 7) and wound closed in two layers (Fig. 8). Patients' weightbearing status was determined by the index procedure.

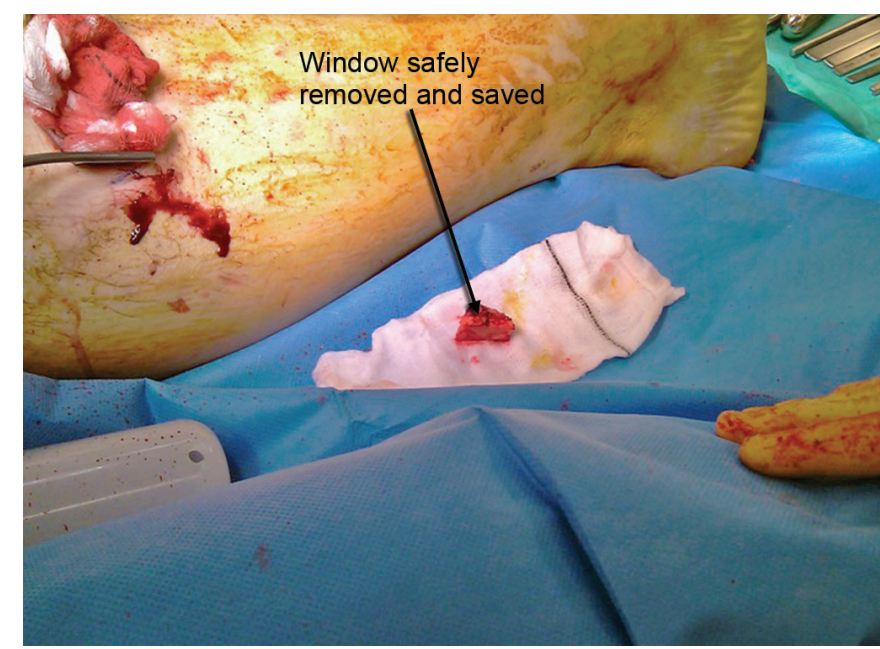

Fig. 4: Cortical window lifted out

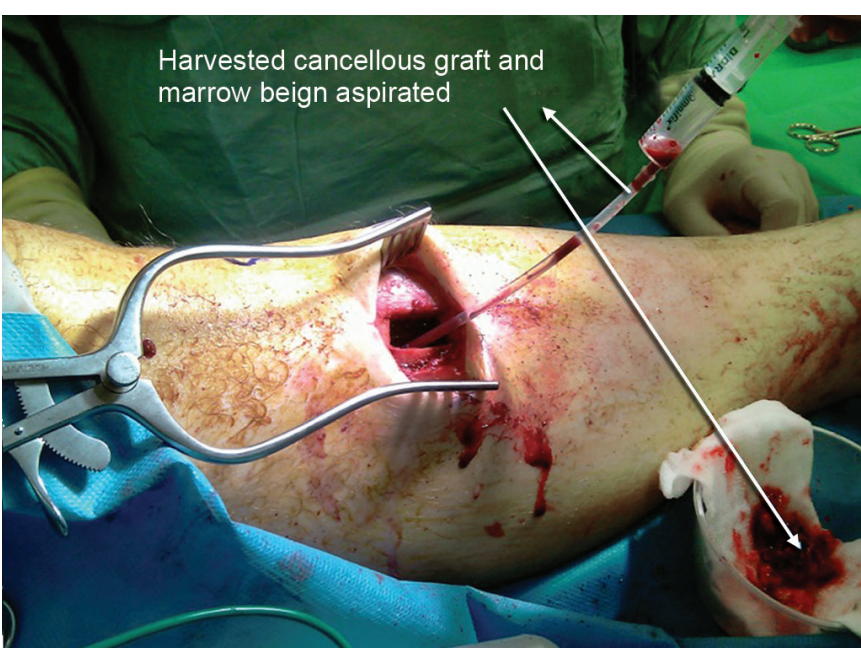

Fig. 6: Marrow harvesting

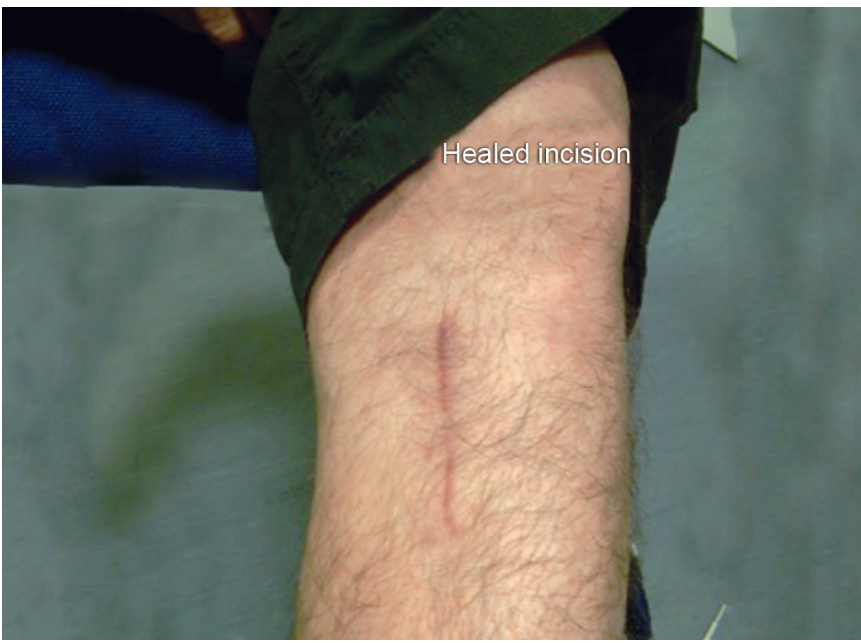

Fig. 8: Healed scar 


\begin{tabular}{ll}
\multicolumn{2}{c}{ Table 1: Index procedures } \\
\hline Index procedures & $\begin{array}{l}\text { Number of } \\
\text { operations done }\end{array}$ \\
\hline Revision first metatarsophalangeal joint fusion & $22(47.8 \%)$ \\
Subtalar joint fusion & $9(19.6 \%)$ \\
Tarsometatarsal joint fusion & $8(17.4 \%)$ \\
Talonavicular joint fusion & $5(10.9 \%)$ \\
Others & 2() \\
\hline
\end{tabular}

\section{MATERIALS AND METHODS}

We conducted a retrospective case series study of all the patients who underwent MPTBGH from 2005 to 2013. Data were collected from case records, electronic patient records, and Picture Archiving and Communicating System. A total of 50 consecutive patients who underwent the procedure were selected. Intraoperatively, quality of bone harvest and technical difficulties in harvesting were recorded.

A total of 45 patients (46 procedures; Table 1) were available for telephone review. Postoperative pain, donor site morbidity, and overall patient satisfaction were noted.

\section{RESULTS}

Mean age was 60.3 years (24-78 years). There were 32 males $(71 \%)$ and 13 females (29\%). All patients had ipsilateral proximal tibia bone graft. The mean duration of follow-up was 14 months (6-36 months).

Intraoperative graft quantity was found to be excellent in all but one case (98\%). We felt graft was not adequate in a 78 -year-old patient. A total of 36 patients $(80 \%)$ were pain free at 6 weeks follow-up; 44 patients $(96 \%)$ were pain free at 3 months. No patient complained of any significant prolonged pain at follow-up.

One patient had a slight uncomfortable numbness at the graft site. There was one fracture through the graft site due to slipping on ice 6 weeks postsurgery.

Patient satisfaction was found to be excellent at the final telephone review. A total of 44 patients $(98 \%)$ were subjectively happy with the procedure and 43 patients (95.6\%) were satisfied with the aesthetic look of the scar; 40 patients $(89 \%)$ said that they would recommend this procedure.

Union rate was $95.7 \%(44 / 46)$. Two patients who underwent 1st metatarsophalangeal joint fusion revision had nonunion, requiring further revision procedures.

Only three cases had postoperative complications. One case was of superficial wound infection, which resolved with antibiotics. Two patients had proximal tibial fracture through the graft area. Both patients had simple mechanical fall. The first patient had the injury at 6 weeks postsurgery and was treated nonoperatively. The second patient had the injury at 4 weeks postsurgery and was treated with open reduction internal fixation (Figs 9 and 10).

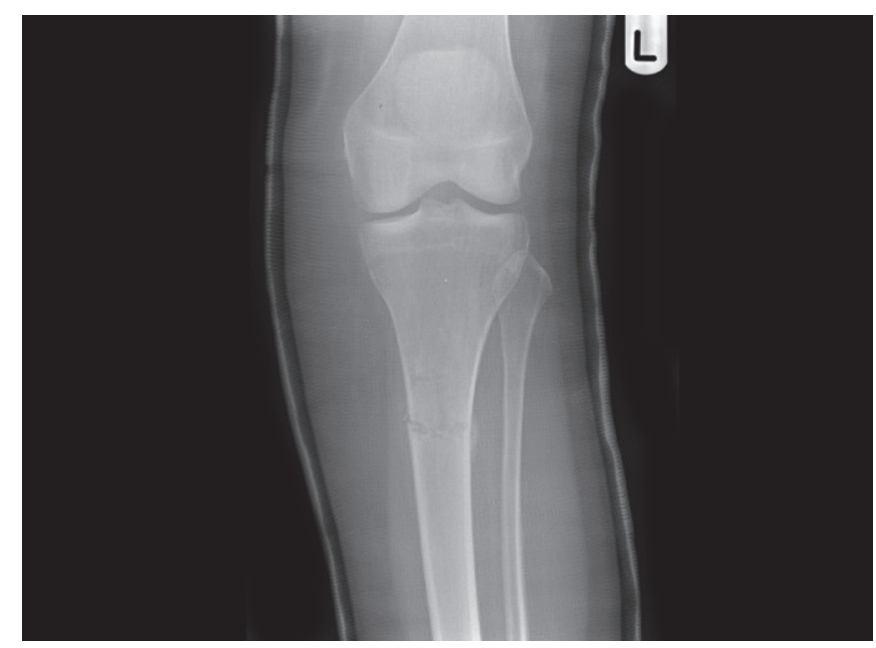

Fig. 9: Tibia fracture: four weeks post-operative

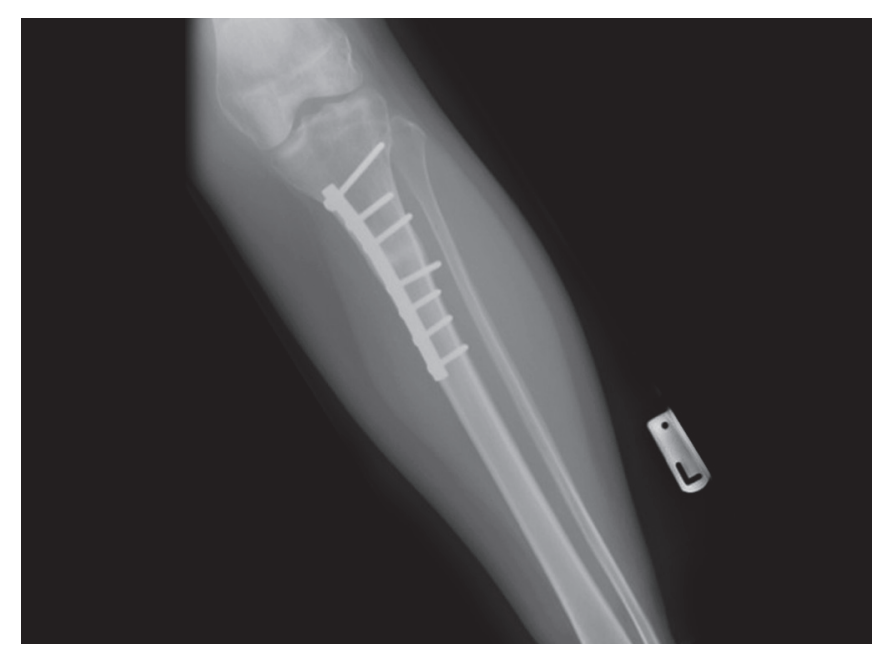

Fig. 10: Tibia fracture treated with open reduction internal fixation

\section{DISCUSSION}

Autogenous cancellous bone graft provides an osteoconductive, osteoinductive, and osteogenic substrate for bone healing. ${ }^{1}$ The proximal tibial metaphysis is a useful site for obtaining autogenous cancellous bone graft and is associated with a low morbidity. ${ }^{2-8} \mathrm{Up}$ to $70 \mathrm{~cm}^{3}$ of cancellous graft can be obtained in young adults with good bone stock and is similar to the volume obtained from iliac crest. ${ }^{1}$ The proximal tibia is a suitable site for bone graft harvest for foot and ankle surgery, because it is within the operative field and is under tourniquet control. There is no need for additional restrictions in weight bearing after this procedure. ${ }^{9}$

Anterior iliac crest has more hematopoietic marrow than any other graft harvest sites and hence is regarded as the gold standard site for bone graft harvest. However, many clinicians recognize considerable disadvantages to using iliac crest for graft harvest during foot and ankle surgery. The most obvious problem is related to its anatomic location, requiring the preparation of a second surgical site. ${ }^{10}$ 
Traditionally, proximal tibial bone graft harvesting (PTBGH) is done by lateral approach. Various authors have described lateral PTBGH in maxillofacial surgery, foot and ankle surgery, and trauma surgery with satisfactory results. However, lateral approach has difficult access and the lateral (interosseous) border of the tibia is vertical. Due to the attachment of iliotibial band and the risk of breaching the joint, the cortical window should be made at or below Gerdy's tubercle by elevating the superior fibers of tibialis anterior muscle. The lateral approach hence breaches the anterior compartment and increases the risk of compartment syndrome. ${ }^{6}$ The branches of the recurrent tibial vessels and nerve coursing through the tibialis anterior muscles are at risk in the lateral approach. Medial approach has fewer serious structures in harm's way compared with lateral approach. However, lateral approach has the benefit of muscle coverage, and hence it might be beneficial for patients with poor wound healing capacity (e.g., diabetics) ${ }^{11}$ With either of these approaches, there is no difference with regard to the clinical results or the complications. ${ }^{6,12}$ Soohoo and Cracchiolo ${ }^{8}$ have quoted 93\% union rate in foot and ankle procedures with PTBGH and confirm difficulties with lateral approach. Vienne et $\mathrm{al}{ }^{12}$ have reported high patient satisfaction with lateral approach and suggested that it can be done under local or locoregional anesthesia.

Alt et $\mathrm{al}^{2}$ have quoted an overall complication rate of $1.9 \%$ with PTBGH. In their series, there were no major complications and immediate postoperative weight bearing did not have any deleterious effects. Geidman et $\mathrm{al}^{5}$ also confirmed similar findings. They reported three incidents of sensory changes at the incision site.

\section{CONCLUSION}

Proximal medial tibial bone graft harvesting is a relatively safe and easy procedure to obtain adequate amounts of autogenous cancellous bone graft for foot and ankle procedures. We believe our study is the largest series using medial approach.

Donor site morbidity is very low. Fusion rate was found to be excellent for foot and ankle procedures. Recovery from the index procedure was not affected and hence is a cost-effective alternative to bone graft substitutes. As with other bone graft donor sites, the quality and quantity of grafts are adversely affected by age above 75 years. We strongly recommend this technique as a gold standard for foot and ankle fusion procedures.

\section{REFERENCES}

1. Myeroff $\mathrm{C}$, Archdeacon M. Autogenous bone graft: donor sites and techniques. J Bone Joint Surg Am 2011 Dec;93(23): 2227-2236.

2. Alt $\mathrm{V}, \mathrm{Nawab} A$, Seligson D. Bone grafting from the proximal tibia. J Trauma 1999 Sep;47(3):555-557.

3. Alt V, Meeder PJ, Seligson D, Schad A, Atienza C Jr. The proximal tibia metaphysis: a reliable donor sight for bone grafting? Clin Orthop Relat Res 2003 Sep;(414):315-321.

4. Chen YC, Chen CH, Chen PL, Huang IY, Shen YS, Chen CM. Donor site morbidity after harvesting of proximal tibia bone. Head Neck 2006 Jun;28(6):496-500.

5. Geidman W, Early JS, Brodsky J. Clinical results of harvesting autogenous cancellous graft from the ipsilateral proximal tibia for use in foot and ankle surgery. Foot Ankle Int 2004 Jul;25(7):451-455.

6. Herford AS, King BJ, Audia F, Becktor J. Medial approach for tibial bone graft: anatomic study and clinical technique. J Oral Maxillofac Surg 2003 Mar;61(3):358-363.

7. O'Keeffe RM Jr, Riemer BL, Butterfield SL. Harvesting of autogenous cancellous bone graft from the proximal tibial metaphysis. A review of 230 cases. J Orthop Trauma 1991 Dec;5(4):469-474.

8. Soohoo NF, Cracchiolo A 3rd. The results of utilizing proximal tibial bone graft in reconstructive procedures of the foot and ankle. Foot Ankle Surg 2008;14(2):62-66.

9. Whitehouse MR, Lankester BJ, Winson IG, Hepple S. Bone graft harvest from the proximal tibia in foot and ankle arthrodesis surgery. Foot Ankle Int 2006 Nov;27(11): 913-916.

10. Winson IG, Higgs A. The use of proximal and distal tibial bone graft in foot and ankle procedures. Foot Ankle Clin 2010 Dec;15(4):553-558.

11. Benninger B, Ross A, Delmarter T. Approaches to proximal tibial bone harvest techniques. J Oral Maxillofac Res 2012 Apr-Jun;3(2):e2.

12. Vienne $\mathrm{P}$, Maurer M, Jankauskas L. Autogenous bone grafting from proximal tibia in elective foot and ankle surgery: an analysis of 43 consecutive patients. Fuss Sprungg 2006;4(1):54-58. 